\title{
Brain Responses during the Anticipation of Dyspnea
}

\author{
M. Cornelia Stoeckel, ${ }^{1}$ Roland W. Esser, ${ }^{1}$ Matthias Gamer, ${ }^{1,2}$ \\ Christian Büchel, ${ }^{1}$ and Andreas von Leupoldtt ${ }^{1,3}$ \\ ${ }^{1}$ Department of Systems Neuroscience, University Medical Center Hamburg-Eppendorf, Martinistraße 52, 20246 Hamburg, Germany \\ ${ }^{2}$ Department of Psychology 1, University of Würzburg, Marcusstraße 9-11, 97070 Würzburg, Germany \\ ${ }^{3}$ Research Group Health Psychology, University of Leuven, Tiensestraat 102, 3000 Leuven, Belgium
}

Correspondence should be addressed to Andreas von Leupoldt; andreas.vonleupoldt@ppw.kuleuven.be

Received 13 January 2016; Revised 6 April 2016; Accepted 15 August 2016

Academic Editor: Daniel W. Wesson

Copyright (C) 2016 M. Cornelia Stoeckel et al. This is an open access article distributed under the Creative Commons Attribution License, which permits unrestricted use, distribution, and reproduction in any medium, provided the original work is properly cited.

\begin{abstract}
Dyspnea is common in many cardiorespiratory diseases. Already the anticipation of this aversive symptom elicits fear in many patients resulting in unfavorable health behaviors such as activity avoidance and sedentary lifestyle. This study investigated brain mechanisms underlying these anticipatory processes. We induced dyspnea using resistive-load breathing in healthy subjects during functional magnetic resonance imaging. Blocks of severe and mild dyspnea alternated, each preceded by anticipation periods. Severe dyspnea activated a network of sensorimotor, cerebellar, and limbic areas. The left insular, parietal opercular, and cerebellar cortices showed increased activation already during dyspnea anticipation. Left insular and parietal opercular cortex showed increased connectivity with right insular and anterior cingulate cortex when severe dyspnea was anticipated, while the cerebellum showed increased connectivity with the amygdala. Notably, insular activation during dyspnea perception was positively correlated with midbrain activation during anticipation. Moreover, anticipatory fear was positively correlated with anticipatory activation in right insular and anterior cingulate cortex. The results demonstrate that dyspnea anticipation activates brain areas involved in dyspnea perception. The involvement of emotion-related areas such as insula, anterior cingulate cortex, and amygdala during dyspnea anticipation most likely reflects anticipatory fear and might underlie the development of unfavorable health behaviors in patients suffering from dyspnea.
\end{abstract}

\section{Introduction}

Dyspnea is the aversive and threatening cardinal symptom in prevalent diseases such as asthma and chronic obstructive pulmonary disease (COPD) and associated with great individual and socioeconomic burden [1]. In chronic respiratory conditions the adequate perception of dyspnea plays a key role as it has a strong influence on health behavior and course of disease. Notably, the perception of dyspnea is not tightly related to objective lung function [2] but is modulated by cognitive and affective factors [3-6].

The few available neuroimaging studies investigating the neural processing of dyspnea [7-14] underline the importance of sensorimotor, cognitive, and emotion-related brain areas. A dual pathway model has been suggested $[15,16]$ with one pathway including ventroposterior thalamic areas and sensorimotor cortices processing the sensorimotor aspects of dyspnea. The second pathway including medial-dorsal thalamic areas, insula, amygdala, and cingulate cortex is believed to process the affective aspects of dyspnea. Of all these areas the paralimbic insula with its implication in interoceptive and emotion-related processing seems to play a key role [4, 11, 16, 17]. Notably, recent studies have demonstrated that negative emotions are related not only to increased perception but also to changes in the neural processing of dyspnea [18].

Patients suffering from chronic dyspnea tend to avoid discomfort by reducing daily-life physical activities. Activity avoidance results in progressive deconditioning, which further increases dyspnea [19]. In particular the fearful anticipation of dyspnea has been hypothesized to lead up to this spiral of decline [20]. Indeed, recent studies demonstrated 
that the anticipation of dyspnea is associated with increased physiological fear responses [21], especially in anxious individuals [22]. Although the fearful anticipation of dyspnea might play a fundamental role for disease progression the underlying brain processes have rarely been studied.

Investigations on the anticipation of pain, a similarly aversive bodily sensation, indicate that pain-sensitive areas are already activated during pain anticipation [23-25]. Moreover, brain activation during pain anticipation predicts and influences the subsequent perception [26] and neural processing of pain [27-29]. Anticipatory changes of brain function in areas with high importance for emotion processing such as insula, anterior cingulate cortex (ACC), amygdala, and midbrain/periaqueductal gray (PAG) were particularly relevant $[26,30,31]$.

Similar mechanisms can be expected for the anticipation and perception of dyspnea. If brain activation during the anticipation of dyspnea would indeed influence and shape subsequent dyspnea perception, this might be particularly relevant for a better understanding of dyspnea avoidance behavior in patients suffering from chronic dyspnea and for the development of tailored treatment strategies.

Therefore, we used functional magnetic resonance imaging (fMRI) to investigate the brain processes underlying the anticipation of resistive-load-induced dyspnea in healthy volunteers. Specifically, we tested the hypotheses that the anticipation of dyspnea is processed in brain areas related to the perception of dyspnea and would involve prominent activations in emotion-related areas [18]. Moreover, we hypothesized that brain activation during dyspnea anticipation would relate to brain activation during subsequent dyspnea perception.

\section{Materials and Methods}

2.1. Participants. We recruited 46 healthy subjects without history of respiratory disease from a large database of genotyped individuals (Table 1). Genotype related differences concerning the neural processing of dyspnea as well as habituation effects have been reported elsewhere [32, 33] while the current analyses specifically focus on anticipatory processes. All data were collected on one day and normal lung function (forced expiratory volume in one second in \% predicted $>80 \%$ ) was confirmed by standard spirometry [34] on the day of the experiment. Written informed consent was obtained prior to the study. The study protocol was approved by the ethics committee of the Medical Association Hamburg (PV3662).

2.2. Apparatus and Respiratory Measurements. Volunteers breathed through a face mask connected with an MRI compatible pneumotachograph (ZAN 600 unit, ZAN Messgeräte $\mathrm{GmbH}$, Oberhulba, Germany). The set-up contained ports for recording of end-tidal $\mathrm{CO}_{2}$ pressure $\left(P_{\mathrm{ET}_{\mathrm{CO}_{2}}}\right)$ and peak inspiratory mouth pressure $\left(P_{I}\right)$ and a two-way nonrebreathing valve. The inspiratory port of the valve was connected to a $2.6 \mathrm{~m}$ tube for the easy manual introduction
TABLE 1: Mean (SD) baseline characteristics of subjects.

\begin{tabular}{|c|c|}
\hline Age (yr) & $28.5(6)$ \\
\hline Sex (female/male), $n$ & $18 / 28$ \\
\hline Weight (kg) & $75.5(12.9)$ \\
\hline Height $(\mathrm{cm})$ & $178.9(9.6)$ \\
\hline Body mass index $\left(\mathrm{kg} / \mathrm{m}^{2}\right)$ & $23.4(2.4)$ \\
\hline $\mathrm{FEV}_{1}(\mathrm{~L})$ & $4.77(0.98)$ \\
\hline $\mathrm{FEV}_{1}(\%$ predicted $)$ & $115.3(12.6)$ \\
\hline FVC (L) & $5.73(1.27)$ \\
\hline FVC (\% predicted) & $117.04(13.14)$ \\
\hline
\end{tabular}

$\mathrm{FEV}_{1}=$ forced expiratory volume in $1 \mathrm{~s}, \mathrm{FVC}=$ forced vital capacity.

and removal of MR-compatible resistive loads in the scanner environment by the experimenter. The free expiratory port prevented rebreathing of $\mathrm{CO}_{2}$. This breathing circuit allowed continuous measurements of respiratory parameters including $P_{\mathrm{ET}_{\mathrm{CO}_{2}}}$, peak inspiratory pressure, tidal volume $\left(V_{T}\right)$, breathing frequency $(f)$, minute ventilation $\left(V_{E}\right)$, and inspiratory time $\left(T_{I}\right)$.

2.3. Induction and Measurement of Perceived Dyspnea. We explained dyspnea to our participants as a sensation of difficult and uncomfortable breathing. In a pretest subjects were placed in a supine position and presented with inspiratory resistive loads of increasing magnitude. Each load was presented for $24 \mathrm{~s}$ and dyspnea intensity subsequently rated on a Borg-scale $(0=$ "not noticeable" to $10=$ "maximally imaginable"). Load magnitude was increased until subjects reliably reported a sensation of "severe" dyspnea (Borg score $\geq 5$ ). The respective load was then used to induce severe dyspnea during scanning (mean load resistance $=$ $2.23 \mathrm{kPa} / \mathrm{L} / \mathrm{s}, \mathrm{SD}=1.18$ ). For the baseline condition of "mild" dyspnea the smallest resistive load that was reliably rated as different from unloaded breathing was used (mean load resistance $=0.25 \mathrm{kPa} / \mathrm{L} / \mathrm{s}, \mathrm{SD}=0.18$ ).

2.4. Instructions. Subjects learned the association of colored cues in the shape of a cross and experimental conditions both during a computer-based standardized instruction outside the scanner and during a short test run within the scanner where subjects were also acquainted with the button response system. Thus, subjects were well familiar with the cue, stimulus association prior to the acquisition of functional MRI data.

2.5. Experimental Protocol. Immediately after pretest and standardized instructions, subjects entered a 3-Tesla TRIOMagnetom Scanner (Siemens, Medical Solutions, Erlangen, Germany) with the face mask tightly fitted. Visual cues and Borg-scales were projected into the scanner bore via a mirror and condition markers were sent to ZAN-system using Presentation software (Neurobehavioral Systems, Inc., Albany, CA). In alternating order, subjects were presented with the visual color-coded cue for either mild or severe dyspnea followed by the respective load (Figure 1). Each 


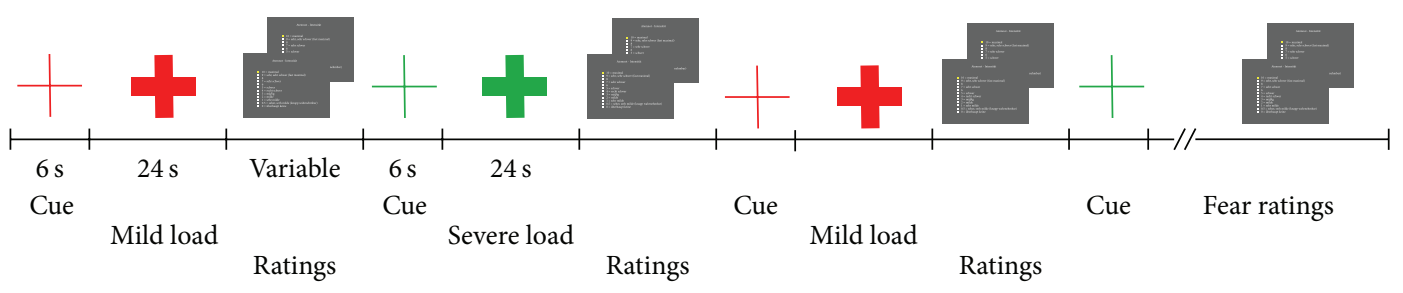

FIGURE 1: MRI task and protocol. In alternating order, subjects were visually cued for either mild or severe dyspnea followed by the respective load. Each load was followed by two Borg rating scales, one on dyspnea intensity and one on dyspnea unpleasantness. The order of the scales was randomized. Each anticipatory cue lasted for $6 \mathrm{~s}$. Then the cue, a thin cross, turned into a solid cross and the load was introduced manually for $24 \mathrm{~s}$. There were ten blocks of mild and severe dyspnea, respectively. The last intensity and unpleasantness ratings were followed by ratings on the average anticipatory fear during the two different cues.

anticipatory period lasted for $6 \mathrm{~s}$. Then the cue, a thin cross, turned into a solid cross and the preselected load was introduced manually for $24 \mathrm{~s}$ as in our previous fMRI studies using similar stimuli [11, 12]. There were 10 blocks of mild and 10 blocks of severe dyspnea. Each load was followed by two Borg rating scales: one on dyspnea intensity and one on dyspnea unpleasantness during the preceding block. The order of the scales was randomized. Following the final dyspnea ratings subjects were presented with two additional Borg-scales asking to indicate the level of fear experienced on average during the cue conditions (anticipation) of mild and severe dyspnea, respectively (Figure 1). Immediately following the brain scan subjects rated the perceived quality of dyspnea on a verbal descriptor list [35] outside the scanner.

2.6. fMRI Data Acquisition. Imaging was performed on a 3Tesla TRIO-Magnetom Scanner (Siemens, Medical Solutions, Erlangen, Germany) using a standard 32-channel headcoil. For each data volume we acquired 48 continuous axial-slices in descending order with $2 \times 2 \mathrm{~mm}$ in-plane resolution, $2 \mathrm{~mm}$ slice thickness, and a $1 \mathrm{~mm}$ gap using $\mathrm{T} 2{ }^{*}$-weighted parallel echoplanar imaging $(\mathrm{TR}=2870 \mathrm{~ms}$, $\mathrm{TE}=25 \mathrm{~ms}$, flip angle $=80^{\circ}$, and field of view $=208 \times$ $208 \mathrm{~mm})$ with GRAPPA acceleration $(R=2)$. Depending on the time spent on ratings subjects needed 13-18 min to complete the protocol. The number of functional scans acquired varied accordingly (275-374 volumes). The first 5 volumes were discarded to allow for T1-saturation. Following fMRI, we acquired a high-resolution T1-weighted structural brain scan using a standard MP-RAGE sequence $(1 \times 1 \times 1 \mathrm{~mm}$ spatial resolution, $\mathrm{TR}=2300 \mathrm{~ms}, \mathrm{TE}=$ $2.98 \mathrm{~ms}$, flip angle $=9^{\circ}$, and field of view $=256 \times 256,240$ slices).

2.7. Data Analysis. Means of intensity, unpleasantness, and anticipatory fear ratings were compared between mild and severe dyspnea conditions using paired $t$-tests. Respiratory parameters for each block and condition were analyzed as dependent variables in separate one-way repeated measures ANOVAs across the four conditions (anticipation mild, mild dyspnea, anticipation severe, severe dyspnea) followed by Bonferroni-corrected paired $t$-tests to further explore significant main effects. Analyses were calculated with SPSS 20.0 software (SPSS Inc., Chicago, IL) using a significance level of $p<0.05$.

All steps of fMRI data preprocessing and statistical analysis were carried out using SPM8 (http://www.fil .ion.ucl.ac.uk/spm/), with the exception of noise-correction, which was carried out using fsl-MELODIC 3.0. From the ten presented blocks of each condition, the first two blocks of each condition (i.e., $2 \times$ anticipation mild, $2 \times$ mild dyspnea, $2 \times$ anticipation severe, and $2 \times$ severe dyspnea) served as adaptation phase and did not enter the analyses. A custom template within standard space was created from the T1 images of all participants using the DARTEL-protocol implemented within SPM8. Functional data were unwarped and realigned using 6-parameter rigid-body transformations. After normalization to the custom-made T1-template using linear and nonlinear transformations, noise was identified based on a probabilistic independent component analysis. Preprocessed data were whitened and projected into a 40dimensional subspace using Principal Component Analysis and further decomposed into sets of vectors that describe signal variation across the temporal domain (time courses) and across the spatial domain (spatial maps) by optimizing for non-Gaussian spatial source distributions [36]. Each component was categorized as either function-related (restingstate networks or paradigm related) or noise-related (e.g., noise due to respiration, cardiac activity, motion, or scanner drifts) by considering the spatial pattern, the time course, and the power distribution following a heuristic described by Kelly et al. [37]. This procedure also controlled for potential effects of $P_{\mathrm{ET}_{\mathrm{CO}}}$ fluctuations on brain activation. Two independent raters showed high interrater agreement (96.6\%, Cohen's Kappa $=0.8$ ). Components consistently identified as noise were filtered out. Noise-corrected functional data were smoothed using an $8 \times 8 \times 8 \mathrm{~mm}$ full-width at half-maximum Gaussian filter.

For statistical analysis data were high-pass filtered with a $128 \mathrm{~s}$ cut-off, while serial correlations were accounted for by using an autoregressive model. Data modeling on the first level involved separate regressors for each condition (cue mild, mild dyspnea, cue severe, severe dyspnea, and ratings) based on the canonical haemodynamic response function implemented in SPM8. Mean BOLD signal intensity of each image was included as regressor of no interest. On the subject-level we contrasted cue severe with cue mild and 
TABLE 2: Group mean (SD) respiratory parameters for conditions of anticipation and perception of dyspnea.

\begin{tabular}{lcccc}
\hline & \multicolumn{2}{c}{ Anticipation of dyspnea } & \multicolumn{2}{c}{ Perception of dyspnea } \\
& Mild & Severe & Mild & $33.82(3.4)$ \\
$P_{\mathrm{ET}_{\mathrm{CO}_{2}}(\mathrm{mmHg})}$ & $33.81(3.46)$ & $33.45(3.4)^{*}$ & $13.69(3.63)$ & $12.72(3.7)^{\dagger}$ \\
$f$ (breaths/min) & $13.81(3.66)$ & $13.75(3.4)$ & $2.27(0.89)$ & $9(3.97)^{\dagger}$ \\
$P_{I}(\mathrm{mbar})$ & $1.11(0.31)$ & $1.05(0.3)$ & $2.06(0.56)$ & $2.43(0.67)^{\dagger}$ \\
$T_{I}(\mathrm{~s})$ & $1.83(0.61)$ & $1.79(0.41)$ & $12.03(2.93)$ & $11.48(2.94)$ \\
$V_{E}(\mathrm{~L} / \mathrm{min})$ & $10.04(2.34)$ & $9.56(2.56)$ & $0.95(0.31)$ & $1(0.39)$ \\
$V_{T}(\mathrm{~L})$ & $0.82(0.35)$ & $0.77(0.3)$ & \\
\hline
\end{tabular}

${ }^{*} p$ corrected $<0.05,{ }^{\dagger} p$ corrected $<0.01$ for anticipation of severe versus anticipation of mild and severe dyspnea versus mild dyspnea, respectively.

$P_{\mathrm{ET}_{\mathrm{CO}_{2}}}=$ end-tidal $\mathrm{CO}_{2}$ pressure; $f=$ breathing frequency; $P_{I}=$ peak inspiratory mouth pressure; $T_{I}=$ inspiratory time; $V_{E}=$ minute ventilation; $V_{T}=$ tidal volume.

severe with mild resistive-load-induced dyspnea to extract brain areas that show more activation during the anticipation and perception of severe versus mild dyspnea, respectively. These two contrast images per subject were then entered into separate random-effects group analyses.

Next, we investigated how dyspnea-related brain areas that also showed activation during dyspnea anticipation interacted with other brain areas during the anticipation of severe as compared to mild dyspnea. For these psychophysiological interactions (PPI [38]), we extracted the average individual time courses from a volume centered on the peak voxel of the (anticipation severe versus anticipation mild) contrast for each of the investigated areas (left insula, parietal operculum, and cerebellum, see results) and used the anticipation of severe versus mild dyspnea as modulatory experimental factor.

Given the assumed prominent role of the insular cortex in processing the affective qualities of perceived dyspnea [11, $16,39,40]$, we investigated the influence of brain activation during dyspnea anticipation on individual average right insular activation during the subsequent perception of resistiveload-induced dyspnea. Individual parameter estimates from the perception contrast (severe dyspnea versus mild dyspnea) served as covariate for the anticipation contrast (anticipation severe dyspnea versus anticipation mild dyspnea).

Finally, we were interested how anticipatory fear is related to anticipatory brain activation by using individual ratings of anticipatory fear (fear during anticipation of severe dyspnea minus fear during anticipation of mild dyspnea) as covariate for the anticipation contrast (anticipation of severe dyspnea versus anticipation of mild dyspnea).

For statistical inference on our results, we used a two-step approach: first, we tested for significantly increased activation throughout the entire brain exceeding a whole-brain familywise error corrected threshold of $p<0.05$ within a cluster of more than 30 contiguous voxels. For the second analysis we chose the following bilateral regions of interest (ROIs): insula, anterior cingulate cortex, amygdala, and a midbrainregion including the PAG. Bilateral masks for insula, anterior cingulate cortex, and amygdala were generated from the automated anatomical labeling (AAL) template described by Tzourio-Mazoyer et al. [41]. AAL was also used to further specify the localization of cerebellar activation. A midbrain

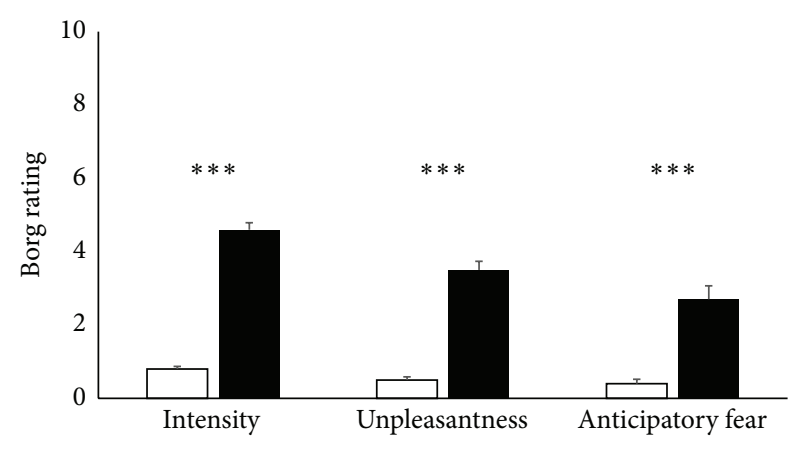

FIGURE 2: Mean (SEM) ratings of perceived intensity, unpleasantness, and anticipatory fear for mild (white) and severe (black) dyspnea, respectively. Increases from mild to severe dyspnea were significant at $p<0.001$. $* * *$ indicates a significance level of $p<$ 0.001 .

ROI centered on PAG was defined using an $8 \mathrm{~mm}$ sphere around the average coordinates for PAG activation reported by Linnman et al. [42]. The selection of these ROIs was based on results of previous studies on the anticipation of aversive stimuli including pain [26, 30,31, 43-45]. Activation within each ROI was considered significant, if exceeding a threshold of $p<0.05$ after family-wise error correction within the ROI.

\section{Results}

3.1. Respiratory Parameters and Dyspnea Ratings. Respiratory parameters showed significant variation across conditions (Table 2). As expected, post hoc $t$-tests showed significantly increased peak inspiratory mouth pressure and inspiratory time, as well as decreased breathing frequency during severe compared to mild dyspnea. During the two anticipation periods subjects showed similar breathing patterns with no significant differences in respiratory parameters except for $P_{\mathrm{ET}_{\mathrm{CO}_{2}}}$, which was slightly lower during the anticipation of severe as compared to mild dyspnea.

Similarly, subjective dyspnea ratings confirmed successful induction of mild and severe dyspnea, respectively (Figure 2). Ratings for intensity and unpleasantness of resistiveload-induced dyspnea were significantly higher during severe 
TABLE 3: Peak coordinates, $t$-statistics, and uncorrected $p$ values for significant brain activations during the perception of severe versus mild dyspnea.

\begin{tabular}{lccccc}
\hline Area & $x$ & $y$ & $z$ & $t$ & $p$ \\
\hline Precentral cortex R & 44 & -8 & 38 & 7.02 & $<0.001^{*}$ \\
& 56 & 4 & 14 & 6.2 & $<0.001^{*}$ \\
Precentral cortex L & -42 & -12 & 40 & 7.73 & $<0.001^{*}$ \\
& -60 & -28 & 18 & 6.7 & $<0.001^{*}$ \\
Postcentral cortex R & 46 & -10 & 36 & 7.3 & $<0.001^{*}$ \\
& 66 & -18 & 20 & 6.3 & $<0.001^{*}$ \\
Postcentral L & -42 & -12 & 34 & 7.11 & $<0.001^{*}$ \\
Supplementary motor area R & 4 & -24 & 62 & 5.75 & $<0.001^{*}$ \\
Supplementary motor area L & -4 & -2 & 60 & 5.46 & $<0.001^{*}$ \\
Parietal operculum (SII) R & 64 & -18 & 20 & 6.31 & $<0.001^{*}$ \\
Parietal operculum (SII) L & -54 & -16 & 20 & 6.41 & $<0.001^{*}$ \\
Insula R & 36 & 6 & 4 & 5.78 & $<0.001^{*}$ \\
Insula L & -36 & 0 & 10 & 5.05 & $<0.001^{\dagger}$ \\
Cerebellar hemisphere R & 14 & -62 & -20 & 5.41 & $<0.001^{*}$ \\
Cerebellar hemisphere L & -26 & -62 & -24 & 6.72 & $<0.001^{*}$ \\
\hline
\end{tabular}

${ }^{*}$ Whole-brain family-wise error corrected $p<0.05$.

${ }^{\dagger}$ Small volume corrected $p$ for respective bilateral ROI $<0.05$.

$x=$ left-right coordinate, $y=$ posterior-anterior coordinate, $z=$ inferiorsuperior coordinate, $\mathrm{R}=$ right hemisphere; $\mathrm{L}=$ left hemisphere.

(mean/SD $=4.6 / 1.3$ and $3.5 / 1.6$, resp.) than during mild dyspnea (mean $/ \mathrm{SD}=0.8 / 0.5$ and $0.5 / 0.6$, resp.). Likewise, the anticipatory fear was significantly stronger for severe as compared to mild dyspnea (mean/SD $=2.7 / 2.5$ and $0.4 / 0.8$, resp.). Verbal descriptor ratings revealed that resistive-loadinduced dyspnea was mainly perceived as "increased work and effort of breathing."

3.2. Functional Imaging Data. When contrasting the perception of severe dyspnea with mild dyspnea the whole-brain analysis confirmed the activation of a bilateral cortical network with activation peaks in pre- and postcentral cortices, SMA, parietal opercular cortex, cerebellum, and right insular cortex (Table 3). The ROI-based analysis yielded additional significant activation of the left insula (Figure 3(a), Table 3).

For anticipation of severe dyspnea versus anticipation of mild dyspnea the whole-brain analysis yielded significant activation that localized to the bilateral occipital pole and the left parietal operculum and cerebellum (Table 4). Further activation was found in bilateral insular cortex, which proved significant for the left anterior insular cortex in the ROI-based analysis (Figure 3(b), Table 4).

Activation during dyspnea anticipation and dyspnea perception showed substantial overlap within the parietal operculum and the cerebellum (6th cerebellar lobule), while insular activation during dyspnea anticipation was more anterior compared to insular activation during dyspnea perception (Figure 3(c)).

Next, we investigated the interactions between anterior insula, parietal operculum, and the 6th cerebellar lobule with other brain areas during the anticipation of dyspnea using PPIs. There were no significant interaction effects in
TABle 4: Peak coordinates $(x, y, z), t$-statistics, and uncorrected $p$ values for significant brain activations during the anticipation of severe versus mild dyspnea.

\begin{tabular}{lccccc}
\hline Area & $x$ & $y$ & $z$ & $t$ & $p$ \\
\hline Occipital pole R & 14 & -96 & 12 & 7.14 & $<0.001^{*}$ \\
Occipital pole L & -8 & -100 & 8 & 8.00 & $<0.001^{*}$ \\
Parietal operculum L & -40 & -32 & 20 & 5.54 & $<0.001^{*}$ \\
Insula L & -30 & 26 & 4 & 4.18 & $<0.001^{\dagger}$ \\
Cerebellar hemisphere L & -28 & -64 & -24 & 5.79 & $<0.001^{*}$ \\
\hline
\end{tabular}

${ }^{*}$ Whole-brain family-wise error corrected $p<0.05$.

${ }^{\dagger}$ Small volume corrected $p$ for respective bilateral ROI $<0.05$.

$x=$ left-right coordinate, $y=$ posterior-anterior coordinate, $z=$ inferiorsuperior coordinate, $\mathrm{R}=$ right hemisphere, and $\mathrm{L}=$ left hemisphere.

the whole-brain analyses. However, the ROI-based approach showed significantly increased interactions of left anterior insula and parietal operculum during dyspnea anticipation with the right insular cortex and the ACC (Figures 4(a) and 4(b)). The left 6 th cerebellar lobule showed a significantly increased interaction with bilateral amygdala (Figure 4(c)).

Furthermore, we looked at the relationship of right insular activation during dyspnea perception with anticipatory brain activation. The ROI-based analysis showed that right insular activation during dyspnea perception was significantly correlated with activation in the midbrain/PAG during the anticipation of severe versus mild dyspnea (Figure 5(a)).

Ratings of anticipatory fear revealed a significant positive correlation with anticipatory brain activation within ACC and right insular cortex in the ROI-based analysis (Figure 5(b)).

\section{Discussion}

The present study investigated brain activations associated with the anticipation and perception of resistive-loadinduced dyspnea in healthy subjects. Our analyses confirmed the involvement of a previously described set of brain areas for the perception of dyspnea [4, 16, 17]. This network included sensorimotor areas (pre- and postcentral gyri, SMA, and parietal operculum), bilateral insular cortex, and the cerebellum. Importantly, within the insular, parietal opercular, and cerebellar cortex activation was already increased during the anticipation of dyspnea. Anticipatory and dyspnea-related activation overlapped within parietal operculum and cerebellum, while activation within the insular cortex was more anterior during anticipation as compared to perception of resistive-load-induced dyspnea. During the anticipation of dyspnea, left anterior insula and parietal operculum showed increased connectivity with ACC and right insular cortex, while the cerebellum showed increased interaction with the bilateral amygdala. Notably, midbrain/PAG activation during dyspnea anticipation correlated with right insular activation during the subsequent perception of dyspnea. Finally, activation in the right insular cortex and ACC during the anticipation of dyspnea showed a significant positive correlation with anticipatory fear. 


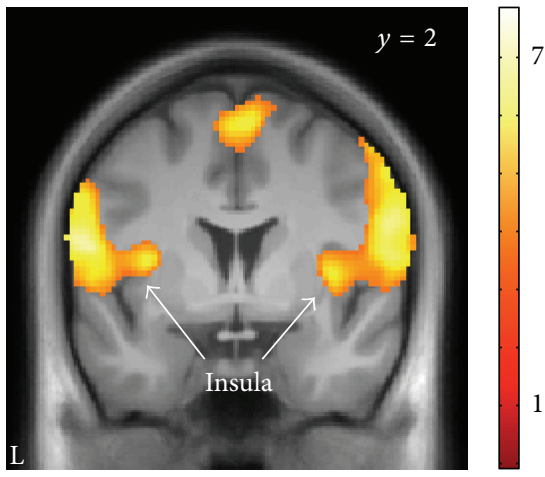

(a)

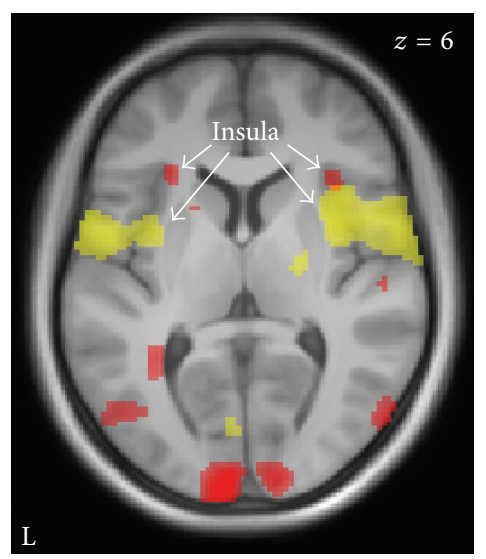

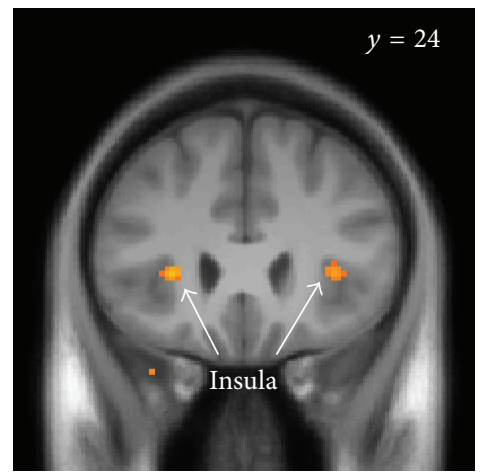

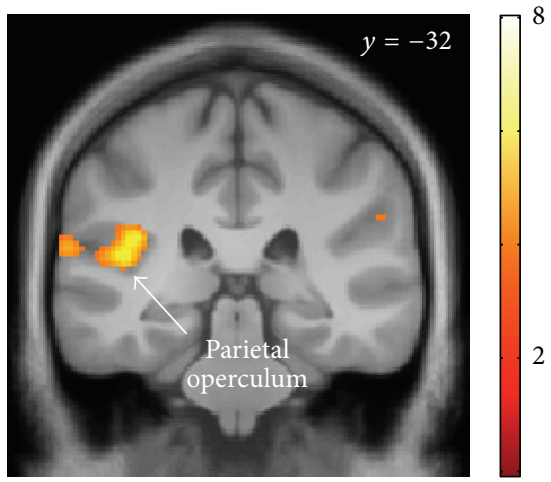

(b)
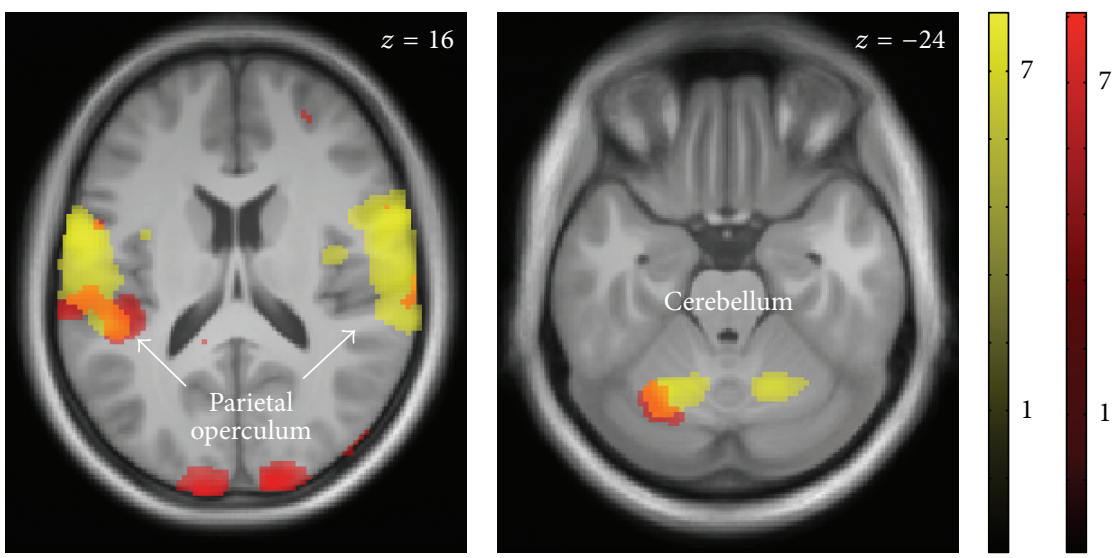

(c)

FIGURE 3: Perception (a) and anticipation (b) of severe versus mild dyspnea activated insular, parietal opercular, and cerebellar cortex. (c) Activation during anticipation (displayed in red) and perception of dyspnea (displayed in yellow) overlapped in the left cerebellum and parietal operculum while insular activation was more anterior during anticipation as compared to perception of dyspnea. Activation patterns are displayed at a threshold of $p<0.05$, corrected for the specific ROI, and superimposed on the group-specific T1-weighted mean image generated by the DARTEL-protocol. $\mathrm{L}=$ left.

Taken together, the present study reveals prominent activation in several emotion-related brain areas during the anticipation of resistive-load-induced dyspnea, which were paralleled by increased anticipatory fear. Thus, both behavioral and functional brain data underline the relevance of affective processes during the anticipation of dyspnea, which in turn partly relate to the subsequent processing of perceived dyspnea.

The present findings converge with previous studies in several ways. First, conditioning studies demonstrated increased physiological fear responses during the anticipation of dyspneic breathing occlusions and hyperventilation including increased startle reflex magnitudes [21, 22]. The present study extends these findings to increased subjective fear reports and the involvement of fear-related brain areas during the anticipation of resistive-load-induced dyspnea.

Second, studies examining the anticipation of other aversive stimuli such as pain [23,30], restricted breathing [40], negative affective pictures $[43,44]$, monetary loss [45], and hyperventilation cues [46] reported comparable activations in emotion-related brain areas as the present study. These included prominent activations in anterior insula, amygdala, anterior cingulate cortex, and PAG. These areas, especially amygdala and insula, have also been described as parts of a salience network for the detection of threatening stimuli [47].

Third, studies comparing anticipation with perception of aversive stimuli such as pain similarly demonstrated overlapping brain activation patterns [23-25, 30]. Furthermore, anticipatory brain activation had an influence on subsequent pain. More specifically, the prestimulus connectivity of anterior insula and midbrain/PAG [26] and anticipatory activation in anterior insula, anterior midcingulate cortex, and amygdala [48] have been found to influence both, brain activation during actual pain perception and behavioral markers of pain.

Fourth, previous studies have linked activation in insula and extended amygdala to the affective unpleasantness of dyspnea [11, 40]. This supports the suggested relevance of these two brain areas for affective responses (e.g., fear) towards upcoming dyspnea, which is further supported by the present correlation between anticipatory fear ratings and anticipatory insular activity.

Finally, overlapping activation for dyspnea anticipation and perception localized to the 6th cerebellar lobule. This 

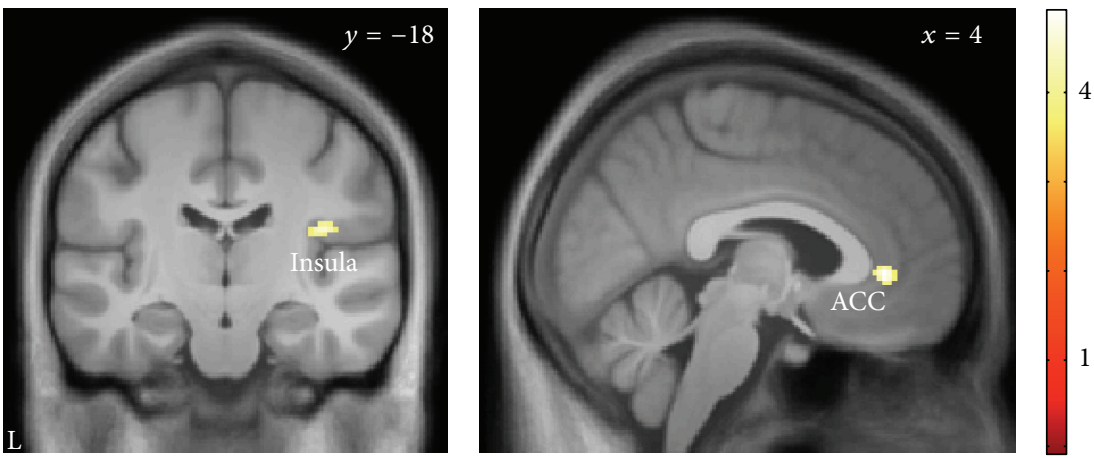

(a)
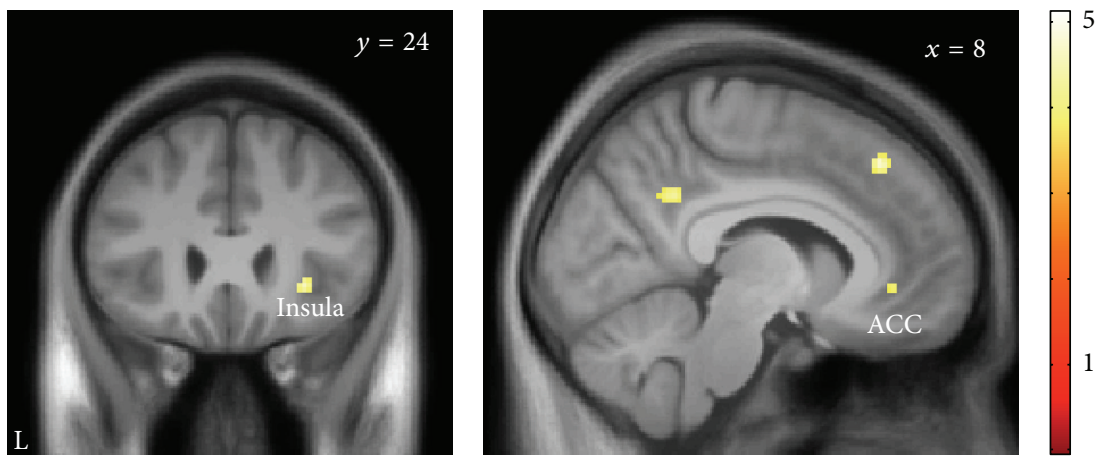

(b)
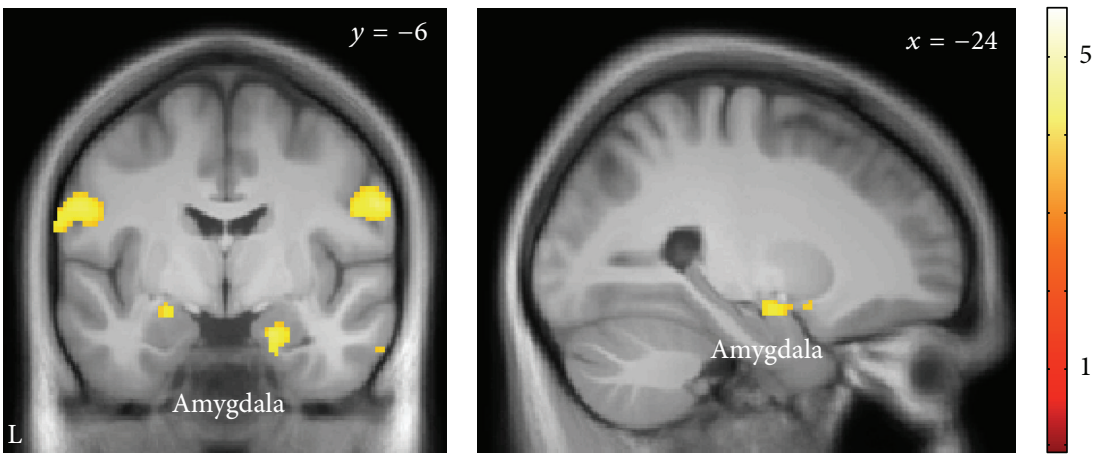

(c)

FIGURE 4: Psychophysiological interactions during the anticipation period. (a) During the anticipation of severe as compared to mild dyspnea the left anterior insular cortex shows significantly increased coactivation with ACC $(x=4, y=38, z=0$; ROI-corrected $p=0.003)$ and right insular cortex $(x=36, y=-18, z=18$; ROI-corrected $p=0.019)$. (b) The left parietal operculum shows increased coactivation with ACC $(x=8, y=40, z=-4$; ROI-corrected $p=0.029)$ and right insular cortex $(x=30, y=26, z=-4$; ROI-corrected $p=0.007)$. (c) The left cerebellar cortex shows increased coactivation with the amygdala, bilaterally $(x=-24, y=-8, z=-14$; ROI-corrected $p=0.011$ and $x=22, y=-4, z=-24$; ROI-corrected $p=0.016$, resp.). The nonsignificant interactions within the rostral cingulate cortex (b) and primary sensorimotor cortex (c) were outside our ROIs and did not reach whole-brain-corrected significance. All interactions are displayed at a threshold of $p<0.05$, corrected for the specific ROI, and superimposed on the group-specific T1-weighted mean image generated by the DARTEL-protocol.

cerebellar subdivision has been shown in neuroimaging and lesion studies to be relevant for emotional processes $[49,50]$ including the processing of different aversive stimuli such as pain and negative affective pictures [51]. Although cerebellar activation has frequently been reported for various dyspneic stimuli $[8,9,52-54]$, its particular contribution to dyspnea perception is only poorly understood. The present observation of anticipatory activity paralleled by strong amygdala interactions is in line with a previously suggested involvement in both, sensorimotor and affective aspects of dyspnea [17].

The present findings suggest a neural correlate for the recently proposed link between anticipatory fear and later avoidance behavior as one underlying cause of negative health outcome in chronic dyspnea [20]. Several clinical studies $[6,55]$ have demonstrated that dyspnea specific 


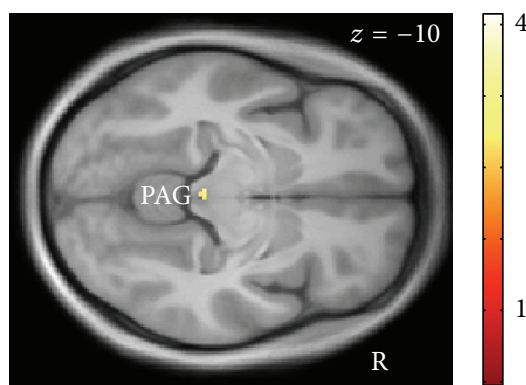

(a)
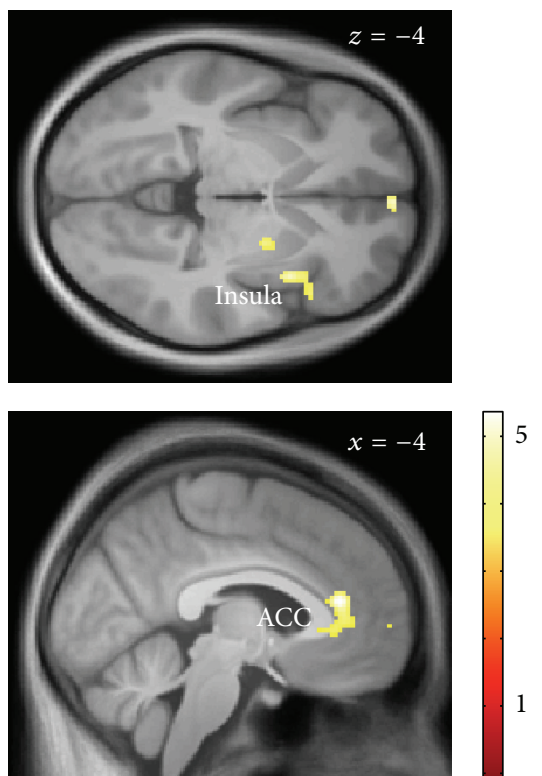

(b)

FIGURE 5: (a) Backward model: right insular activation during dyspnea perception is significantly correlated with midbrain/PAG activation during dyspnea anticipation $(x=-2, y=-30$, $z=-10$; ROI-corrected $p=0.026$ ). (b) Anticipatory fear is significantly correlated with increased brain activation during dyspnea anticipation in the ACC $(x=-4, y=36, z=14$; ROIcorrected $p=0.001)$ and right insular cortex $(x=38, y=12$, $z=-4$; ROI-corrected $p=0.007)$. The nonsignificant correlations with the putamen and medial prefrontal cortex (b) were outside our ROIs and did not reach whole-brain-corrected significance. All correlations are displayed at a threshold of $p<0.05$, corrected for the specific ROI, and superimposed on the group-specific T1weighted mean image generated by the DARTEL-protocol.

fears or worries about physical exercise are related to worse performance in exercise tests and worse outcome of pulmonary rehabilitation in patients with COPD. These findings suggest that irrespective of disease severity anticipatory fear of dyspnea leads to unfavorable health behavior such as avoidance of higher exercise levels. A similar connection between fear of bodily symptoms (e.g., lower back pain), avoidance behavior, and subsequent negative course of disease has already been established in the fear avoidance model of pain [56]. Notably, pain research has already demonstrated the beneficial effects of cognitive behavioral treatments for reducing anticipatory fear of pain and improving the course of disease [57].

Although dyspnea and pain are processed by distinct neural pathways, similarities concerning the emotion-related processes during the anticipation of aversive bodily sensation can be assumed [12]. Therefore, adapting these treatments that have been proven successful in chronic pain to the treatment of anticipatory fear of dyspnea in patients suffering from chronic dyspnea seems promising [58]. Findings of reduced gray matter volume in, for example, ACC and amygdala, in patients suffering from chronic obstructive pulmonary disease [59] provide first evidence that chronic dyspnea indeed impacts the neural structure of emotionrelated areas, potentially related to anticipatory fear.

The following limitations of the present study should be kept in mind: To keep the contingency of anticipation and dyspnea periods, anticipatory fear was not assessed immediately after each anticipation cue. A prompt rating after the anticipation period would certainly allow a more precise assessment of anticipatory fear and would also reflect potential fluctuations over time. Next, we investigated resistiveload-induced dyspnea causing a sensation of increased work and effort of breathing. Thus, our results cannot be generalized to other qualities of dyspnea such as air hunger and chest tightness. Furthermore, our data on predominantly young healthy subjects cannot be generalized to older subjects in general and subjects suffering from chronic dyspnea in particular. Therefore, further research is needed to investigate whether anticipatory fear and respective brain activation patterns within our experimental setting are suitable approximations to understand avoidance behavior in everyday life including reduced physical activity in patients suffering from chronic dyspnea. Respective findings would open a new avenue to behavioral training aimed at reducing anticipatory fear of dyspnea in the treatment of chronic dyspnea.

\section{Conclusions}

Dyspnea anticipation and perception share a similar set of brain areas. Furthermore, anticipatory midbrain/PAG activation was associated with subsequent dyspnea-related activation of the insular cortex. During dyspnea anticipation the prominent involvement of emotion-related areas such as insula, ACC, and amygdala is suggested as potential correlate of anticipatory fear of dyspnea, which might underlie the development of unfavorable health behaviors in patients suffering from dyspnea.

\section{Competing Interests}

The authors declare that there is no conflict of interests regarding the publication of this paper.

\section{Acknowledgments}

This study was supported by grants from the German Research Foundation to Dr. von Leupoldt (DFG LE 1843/92, 10-1, and 10-3). The authors wish to thank Timo Kraemer, 
Katrin Mueller, and Kathrin Wendt for technical assistance with data acquisition and all volunteers for participation in this study.

\section{References}

[1] M. B. Parshall, R. M. Schwartzstein, L. Adams et al., "An official American thoracic society statement: update on the mechanisms, assessment, and management of dyspnea," American Journal of Respiratory and Critical Care Medicine, vol. 185, no. 4, pp. 435-452, 2012.

[2] M. A. Spruit, S. J. Singh, C. Garvey et al., "An official American Thoracic Society/European Respiratory Society statement: key concepts and advances in pulmonary rehabilitation," American Journal of Respiratory and Critical Care Medicine, vol. 188, no. 8, pp. e13-e64, 2013.

[3] R. W. Lansing, R. H. Gracely, and R. B. Banzett, "The multiple dimensions of dyspnea: review and hypotheses," Respiratory Physiology \& Neurobiology, vol. 167, no. 1, pp. 53-60, 2009.

[4] M. Herigstad, A. Hayen, K. Wiech, and K. T. S. Pattinson, "Dyspnoea and the brain," Respiratory Medicine, vol. 105, no. 6, pp. 809-817, 2011.

[5] T. Janssens, G. Verleden, S. De Peuter, I. Van Diest, and O. Van den Bergh, "Inaccurate perception of asthma symptoms: a cognitive-affective framework and implications for asthma treatment," Clinical Psychology Review, vol. 29, no. 4, pp. 317327, 2009.

[6] T. Janssens, S. De Peuter, L. Stans et al., "Dyspnea perception in COPD: association between anxiety, dyspnea-related fear, and dyspnea in a pulmonary rehabilitation program," Chest, vol. 140, no. 3, pp. 618-625, 2011.

[7] R. B. Banzett, H. E. Mulnier, K. Murphy, S. D. Rosen, R. J. S. Wise, and L. Adams, "Breathlessness in humans activates insular cortex," NeuroReport, vol. 11, no. 10, pp. 2117-2120, 2000.

[8] C. Peiffer, J.-B. Poline, L. Thivard, M. Aubier, and Y. Samson, "Neural substrates for the perception of acutely induced dyspnea," American Journal of Respiratory and Critical Care Medicine, vol. 163, no. 4, pp. 951-957, 2001.

[9] K. C. Evans, R. B. Banzett, L. Adams, L. McKay, R. S. J. Frackowiak, and D. R. Corfield, "Bold fMRI identifies limbic, paralimbic, and cerebellar activation during air hunger," Journal of Neurophysiology, vol. 88, no. 3, pp. 1500-1511, 2002.

[10] L. C. McKay, L. Adams, R. S. J. Frackowiak, and D. R. Corfield, "A bilateral cortico-bulbar network associated with breath holding in humans, determined by functional magnetic resonance imaging," NeuroImage, vol. 40, no. 4, pp. 1824-1832, 2008.

[11] A. von Leupoldt, T. Sommer, S. Kegat et al., "The unpleasantness of perceived dyspnea is processed in the anterior insula and amygdala," American Journal of Respiratory and Critical Care Medicine, vol. 177, no. 9, pp. 1026-1032, 2008.

[12] A. von Leupoldt, T. Sommer, S. Kegat et al., "Dyspnea and pain share emotion-related brain network," NeuroImage, vol. 48, no. 1, pp. 200-206, 2009.

[13] K. T. S. Pattinson, R. J. Governo, B. J. MacIntosh et al., "Opioids depress cortical centers responsible for the volitional control of respiration," The Journal of Neuroscience, vol. 29, no. 25, pp. 8177-8186, 2009.

[14] M. Raux, L. Tyvaert, M. Ferreira et al., "Functional magnetic resonance imaging suggests automatization of the cortical response to inspiratory threshold loading in humans," Respiratory Physiology \& Neurobiology, vol. 189, no. 3, pp. 571-580, 2013.
[15] A. von Leupoldt and B. Dahme, "Cortical substrates for the perception of dyspnea," Chest, vol. 128, no. 1, pp. 345-354, 2005.

[16] P. W. Davenport and A. Vovk, "Cortical and subcortical central neural pathways in respiratory sensations," Respiratory Physiology \& Neurobiology, vol. 167, no. 1, pp. 72-86, 2009.

[17] K. C. Evans, "Cortico-limbic circuitry and the airways: insights from functional neuroimaging of respiratory afferents and efferents," Biological Psychology, vol. 84, no. 1, pp. 13-25, 2010.

[18] A. Von Leupoldt, P.-Y. S. Chan, R. W. Esser, and P. W. Davenport, "Emotions and neural processing of respiratory sensations investigated with respiratory-related evoked potentials," Psychosomatic Medicine, vol. 75, no. 3, pp. 244-252, 2013.

[19] J. Z. Reardon, S. C. Lareau, and R. ZuWallack, "Functional status and quality of life in chronic obstructive pulmonary disease," The American Journal of Medicine, vol. 119, no. 10, supplement 1 , pp. 32-37, 2006.

[20] A. Hayen, M. Herigstad, and K. T. S. Pattinson, "Understanding dyspnea as a complex individual experience," Maturitas, vol. 76, no. 1, pp. 45-50, 2013.

[21] M. Pappens, E. Smets, D. Vansteenwegen, O. Van Den Bergh, and I. Van Diest, "Learning to fear suffocation: a new paradigm for interoceptive fear conditioning," Psychophysiology, vol. 49, no. 6, pp. 821-828, 2012.

[22] C. A. Melzig, J. M. Michalowski, K. Holtz, and A. O. Hamm, "Anticipation of interoceptive threat in highly anxiety sensitive persons," Behaviour Research and Therapy, vol. 46, no. 10, pp. 1126-1134, 2008.

[23] C. A. Porro, P. Baraldi, G. Pagnoni et al., "Does anticipation of pain affect cortical nociceptive systems?" The Journal of Neuroscience, vol. 22, no. 8, pp. 3206-3214, 2002.

[24] T. Koyama, J. G. McHaffie, P. J. Laurienti, and R. C. Coghill, "The subjective experience of pain: where expectations become reality," Proceedings of the National Academy of Sciences of the United States of America, vol. 102, no. 36, pp. 12950-12955, 2005.

[25] R. G. Wise, B. J. Lujan, P. Schweinhardt, G. D. Peskett, R. Rogers, and I. Tracey, "The anxiolytic effects of midazolam during anticipation to pain revealed using fMRI," Magnetic Resonance Imaging, vol. 25, no. 6, pp. 801-810, 2007.

[26] M. Ploner, M. C. Lee, K. Wiech, U. Bingel, and I. Tracey, "Prestimulus functional connectivity determines pain perception in humans," Proceedings of the National Academy of Sciences of the United States of America, vol. 107, no. 1, pp. 355-360, 2010.

[27] M. Fairhurst, K. Wiech, P. Dunckley, and I. Tracey, "Anticipatory brainstem activity predicts neural processing of pain in humans," Pain, vol. 128, no. 1-2, pp. 101-110, 2007.

[28] K. Wiech, C.-S. Lin, K. H. Brodersen, U. Bingel, M. Ploner, and I. Tracey, "Anterior insula integrates information about salience into perceptual decisions about pain," The Journal of Neuroscience, vol. 30, no. 48, pp. 16324-16331, 2010.

[29] K. H. Brodersen, K. Wiech, E. I. Lomakina et al., "Decoding the perception of pain from fMRI using multivariate pattern analysis," NeuroImage, vol. 63, no. 3, pp. 1162-1170, 2012.

[30] A. Ploghaus, I. Tracey, J. S. Gati et al., "Dissociating pain from its anticipation in the human brain," Science, vol. 284, no. 5422, pp. 1979-1981, 1999.

[31] I. A. Strigo, A. N. Simmons, S. C. Matthews, A. D. Craig, and M. P. Paulus, "Association of major depressive disorder with altered functional brain response during anticipation and processing of heat pain," Archives of General Psychiatry, vol. 65, no. 11, pp. 1275-1284, 2008. 
[32] M. C. Stoeckel, R. W. Esser, M. Gamer, R. Kalisch, C. Büchel, and A. von Leupoldt, "Amygdala response to anticipation of dyspnea is modulated by 5-HTTLPR genotype," Psychophysiology, vol. 52, no. 7, pp. 973-976, 2015.

[33] M. C. Stoeckel, R. W. Esser, M. Gamer, C. Büchel, and A. von Leupoldt, "Brain mechanisms of short-term habituation and sensitization toward dyspnea," Frontiers in Psychology, vol. 6, article 748, 2015.

[34] M. R. Miller, J. Hankinson, V. Brusasco et al., "Standardisation of spirometry," The European Respiratory Journal, vol. 26, no. 2, pp. 319-338, 2005.

[35] A. von Leupoldt, S. Balewski, S. Petersen et al., "Verbal descriptors of dyspnea in patients with COPD at different intensity levels of dyspnea," Chest, vol. 132, no. 1, pp. 141-147, 2007.

[36] C. F. Beckmann and S. M. Smith, "Probabilistic independent component analysis for functional magnetic resonance imaging," IEEE Transactions on Medical Imaging, vol. 23, no. 2, pp. 137-152, 2004.

[37] R. E. Kelly, G. S. Alexopoulos, Z. Wang et al., "Visual inspection of independent components: defining a procedure for artifact removal from fMRI data," Journal of Neuroscience Methods, vol. 189, no. 2, pp. 233-245, 2010.

[38] K. J. Friston, C. Buechel, G. R. Fink, J. Morris, E. Rolls, and R. J. Dolan, "Psychophysiological and modulatory interactions in neuroimaging," NeuroImage, vol. 6, no. 3, pp. 218-229, 1997.

[39] A. von Leupoldt, T. Sommer, S. Kegat et al., "Down-regulation of insular cortex responses to dyspnea and pain in asthma," American Journal of Respiratory and Critical Care Medicine, vol. 180, no. 3, pp. 232-238, 2009.

[40] M. P. Paulus, T. Flagan, A. N. Simmons et al., "Subjecting elite athletes to inspiratory breathing load reveals behavioral and neural signatures of optimal performers in extreme environments," PLoS ONE, vol. 7, no. 1, Article ID e29394, 2012.

[41] N. Tzourio-Mazoyer, B. Landeau, D. Papathanassiou et al., "Automated anatomical labeling of activations in SPM using a macroscopic anatomical parcellation of the MNI MRI singlesubject brain," NeuroImage, vol. 15, no. 1, pp. 273-289, 2002.

[42] C. Linnman, E. A. Moulton, G. Barmettler, L. Becerra, and D. Borsook, "Neuroimaging of the periaqueductal gray: state of the field," NeuroImage, vol. 60, no. 1, pp. 505-522, 2012.

[43] K. L. Mackiewicz, I. Sarinopoulos, K. L. Cleven, and J. B. Nitschke, "The effect of anticipation and the specificity of sex differences for amygdala and hippocampus function in emotional memory," Proceedings of the National Academy of Sciences of the United States of America, vol. 103, no. 38, pp. 14200-14205, 2006.

[44] J. B. Nitschke, I. Sarinopoulos, K. L. MacKiewicz, H. S. Schaefer, and R. J. Davidson, "Functional neuroanatomy of aversion and its anticipation," NeuroImage, vol. 29, no. 1, pp. 106-116, 2006.

[45] G. R. Samanez-Larkin, N. G. Hollon, L. L. Carstensen, and B. Knutson, "Individual differences in insular sensitivity during loss anticipation predict avoidance learning," Psychological Science, vol. 19, no. 4, pp. 320-323, 2008.

[46] K. Holtz, C. A. Pané-Farré, J. Wendt, M. Lotze, and A. O. Hamm, "Brain activation during anticipation of interoceptive threat," NeuroImage, vol. 61, no. 4, pp. 857-865, 2012.

[47] W. W. Seeley, V. Menon, A. F. Schatzberg et al., "Dissociable intrinsic connectivity networks for salience processing and executive control," The Journal of Neuroscience, vol. 27, no. 9, pp. 2349-2356, 2007.
[48] A. Lutz, D. R. McFarlin, D. M. Perlman, T. V. Salomons, and R. J. Davidson, "Altered anterior insula activation during anticipation and experience of painful stimuli in expert meditators," NeuroImage, vol. 64, no. 1, pp. 538-546, 2013.

[49] F. Bodranghien, A. Bastian, C. Casali et al., "Consensus paper: revisiting the symptoms and signs of cerebellar syndrome," Cerebellum, vol. 15, no. 3, pp. 369-391, 2016.

[50] C. J. Stoodley and J. D. Schmahmann, "Functional topography in the human cerebellum: a meta-analysis of neuroimaging studies," NeuroImage, vol. 44, no. 2, pp. 489-501, 2009.

[51] E. A. Moulton, I. Elman, G. Pendse, J. Schmahmann, L. Becerra, and D. Borsook, "Aversion-related circuitry in the cerebellum: responses to noxious heat and unpleasant images," The Journal of Neuroscience, vol. 31, no. 10, pp. 3795-3804, 2011.

[52] L. M. Parsons, G. Egan, M. Liotti et al., "Neuroimaging evidence implicating cerebellum in the experience of hypercapnia and hunger for air," Proceedings of the National Academy of Sciences of the United States of America, vol. 98, no. 4, pp. 2041-2046, 2001.

[53] V. G. Macefield, S. C. Gandevia, and L. A. Henderson, "Neural sites involved in the sustained increase in muscle sympathetic nerve activity induced by inspiratory capacity apnea: a fMRI study," Journal of Applied Physiology, vol. 100, no. 1, pp. 266-273, 2006.

[54] A. P. Binks, K. C. Evans, J. D. Reed, S. H. Moosavi, and R. B. Banzett, "The time-course of cortico-limbic neural responses to air hunger," Respiratory Physiology and Neurobiology, vol. 204, pp. 78-85, 2014.

[55] M. J. Fischer, M. Scharloo, J. Abbink et al., "Concerns about exercise are related to walk test results in pulmonary rehabilitation for patients with COPD," International Journal of Behavioral Medicine, vol. 19, no. 1, pp. 39-47, 2012.

[56] M. Leeuw, M. E. J. B. Goossens, S. J. Linton, G. Crombez, K. Boersma, and J. W. S. Vlaeyen, "The fear-avoidance model of musculoskeletal pain: current state of scientific evidence," Journal of Behavioral Medicine, vol. 30, no. 1, pp. 77-94, 2007.

[57] J. R. de Jong, J. W. S. Vlaeyen, M. van Eijsden, C. Loo, and P. Onghena, "Reduction of pain-related fear and increased function and participation in work-related upper extremity pain (WRUEP): effects of exposure in vivo," Pain, vol. 153, no. 10, pp. 2109-2118, 2012.

[58] A. von Leupoldt and T. Janssens, "Could targeting disease specific fear and anxiety improve COPD outcomes?" Expert Review of Respiratory Medicine, vol. 10, no. 8, pp. 835-837, 2016.

[59] R. W. Esser, M. C. Stoeckel, A. Kirsten et al., "Structural brain changes in patients with COPD," Chest, vol. 149, no. 2, pp. 426434, 2016. 

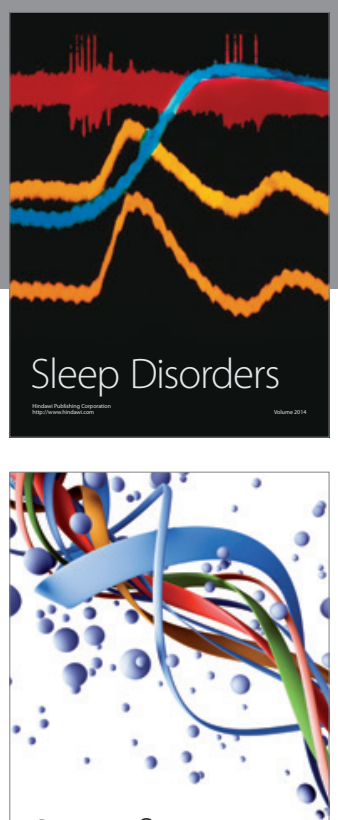

Scientifica
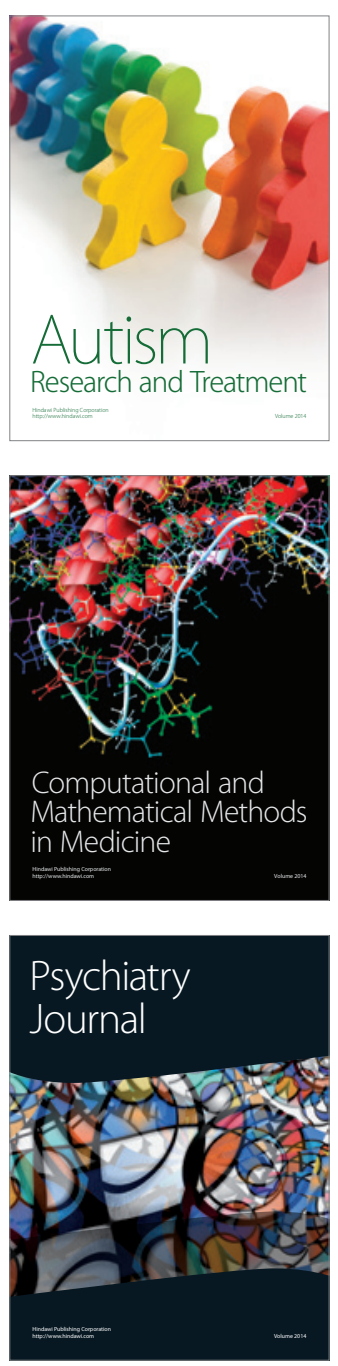
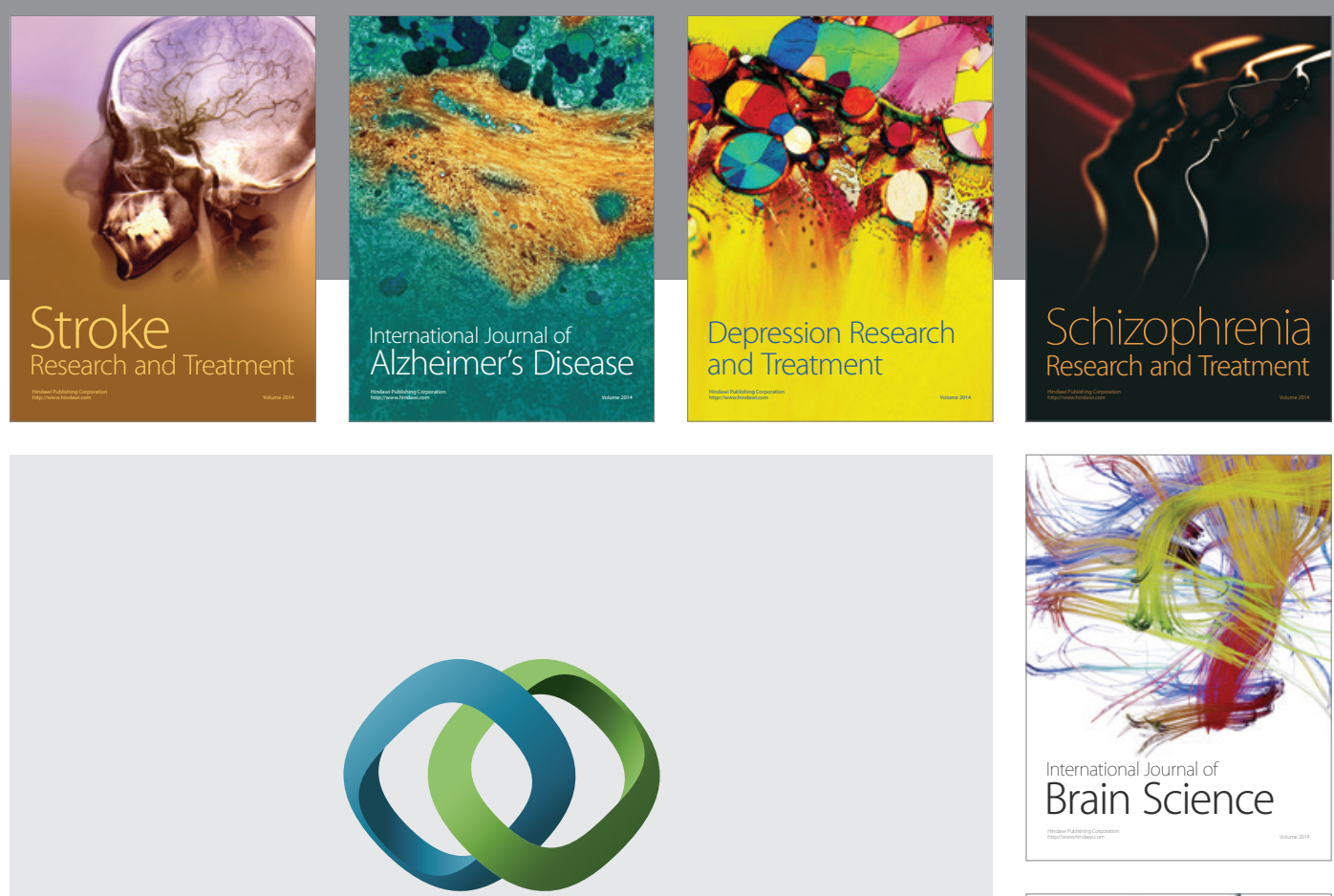

\section{Hindawi}

Submit your manuscripts at

http://www.hindawi.com
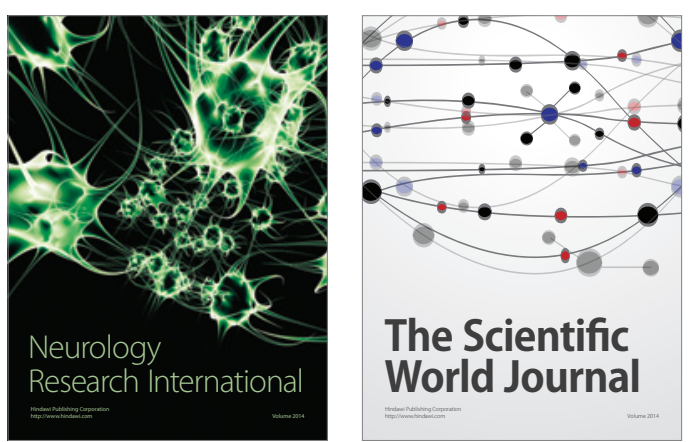

The Scientific World Journal

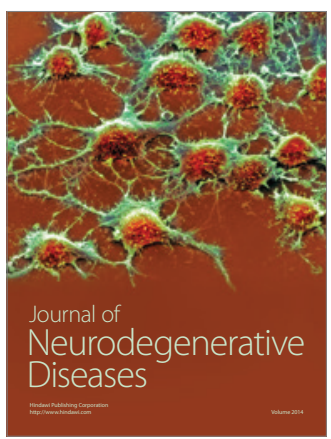

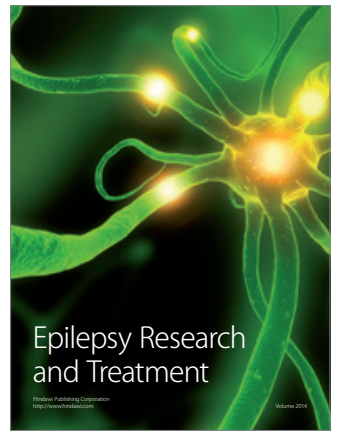

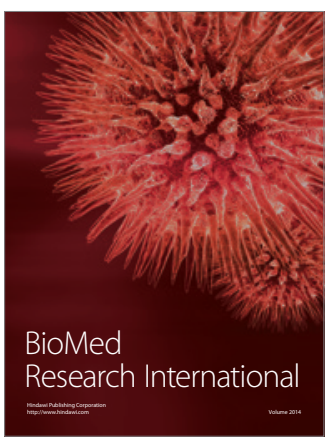

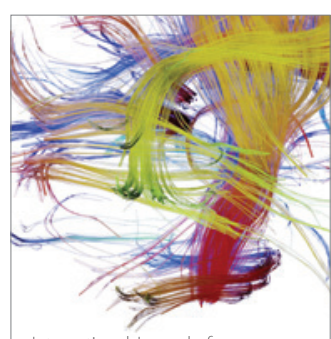

Brain Science

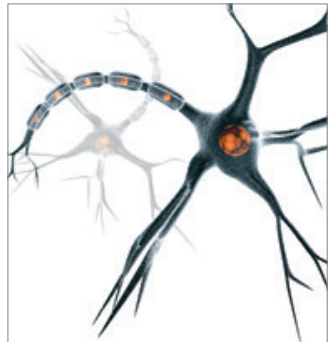

Neural Plasticity
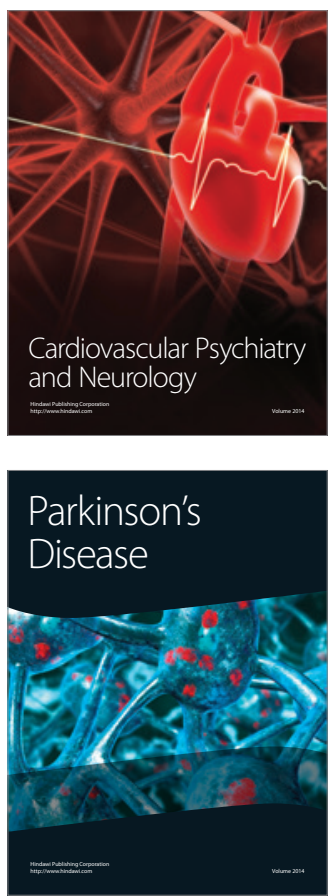